\title{
Design with uncertain qualitative variables under imperfect knowledge
}

\author{
D J Pons ${ }^{1^{*}}$ and $\mathrm{J}$ K Raine ${ }^{2}$ \\ ${ }^{1}$ School of Engineering, Christchurch Polytechnic Institute of Technology, Christchurch, New Zealand \\ ${ }^{2}$ Department of Mechanical Engineering, University of Canterbury, Christchurch, New Zealand
}

Abstract: To support early design and design under risk, it is necessary to have methodologies to process the various forms of uncertainties. Three independent dimensions of uncertainty are identified in the paper as certainty of analysis (epistemic uncertainty), random variability (stochastic variability and design indecision), and type of variable. The type of variable is further categorised into six scales that are broadly grouped into quantitative and qualitative. Common engineering modelling tools used for design do not operate well on combinations of random variables, qualitative variables, and imperfect knowledge. The hypothesis of this paper is that a modelling system could be developed to accommodate the multiple types of uncertainty that can exist during engineering design. This is worth doing as accommodating design uncertainty is an important part of risk management in engineering. The paper then proceeds to describe the way in which the design for system integrity (DSI) methodology meets these objectives. DSI may be used to create models with uncertain variables (including textual and non-ordered), given subjective and imperfect knowledge (including uncertain opinion). Consequently, DSI supports risk management in engineering design.

Keywords: design, risk, uncertainty, probability, qualitative, subjective

\footnotetext{
* Corresponding author: School of Engineering, Christchurch Polytechnic Institute of Technology, PO Box 540, Christchurch, 8015, New Zealand.

Manuscript of 1 May 2003, revised 2 September 2003, revised 8 November 2003, submitted to Journal of Engineering Manufacture (Proceedings of the Institution of Mechanical Engineers Part B).
} 


\section{INTRODUCTION}

A characteristic of early design is the existence of imperfect knowledge and uncertain variables. However, conventional engineering design tools tend to be deterministic: they apply well defined mathematical relationships to variables that are known with certainty, with reserve or safety factors being used to compensate for the uncertainty that is expected to exist. These approaches have proved practical for detailed design operating within an existing body of knowledge. However, determinism can be problematic at early design stages, or when exploring new design envelopes, or when the design has a significant risk element.

The hypothesis of this paper is that a modelling system could be developed to accommodate the multiple types of uncertainty that can exist during engineering design. This is worth doing as accommodating design uncertainty is an important part of risk management in engineering. The work is derived from the Design for System Integrity (DSI) methodology [1], and previous papers in this journal described the background [2], and a method for processing random quantitative variables through mathematically explicit models [3]. The present paper extends the scope to the processing of qualitative variables through imperfectly understood systems, a situation typical of design under risk. The paper first establishes several dimensions of uncertainty, then identifies existing methodologies for qualitative variables, and finally describes the DSI approach. 


\section{DIMENSIONS OF UNCERTAINTY}

It is suggested that different forms of uncertainty exist in engineering design, expressible by three orthogonal dimensions. These are (a) the epistemic uncertainty, (b) the stochastic uncertainty, and (c) the abstraction uncertainty. These dimensions are shown in Figure 1, and clarified below.

Epistemic uncertainty (or certainty of analysis') describes the degree to which the body of knowledge can adequately predict system behaviour from input variables. The range, in decreasing order of completeness, covers: explicit functions (axioms, mathematical equations), correlation (statistical regression), logic (Boolean) \& rules, and opinion (which can be partitioned into knowledge and confidence [4], or support and plausibility [5]). This range has been split into 'objective' and 'subjective' knowledge, with the separation within 'logic \& rules'.

The stochastic uncertainty (or random variability) dimension describes the uncertainty about the precise value that the variable will take. It ranges from deterministic (no uncertainty), probabilistic (or stochastic, based on the likelihood of mutually exclusive events), interval ('possibilistic' fuzzy theory, set membership), to subjective probability (statements such as 'likely'). There are several sub-types of probabilistic variable including a full probability distribution, histogram, decision tree, or single probability (e.g. success vs failure). Also, there are several sub-types of interval variable including simple substitution of different values ('what-if-analysis'), interval analysis 
(all values across a range are considered equally likely to occur), and a fuzzy set (different likelihoods across the range). Design indecision (the designer has not yet decided on the value for a parameter) has been included as a type of random variable, although it is acknowledged that it is an interval rather than strictly random variable as the selection will not necessarily be based on the mean or other probability statistics.

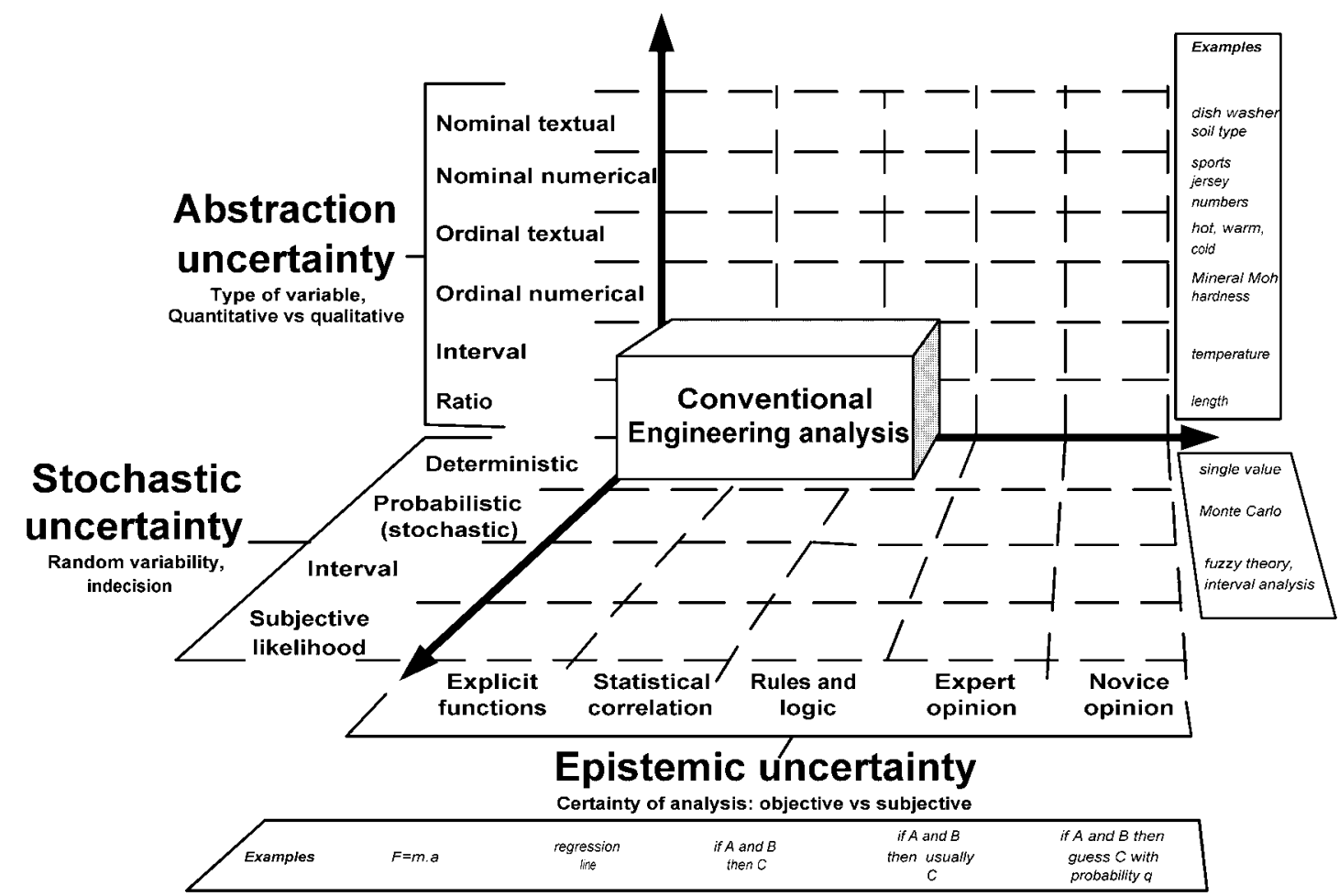

Figure 1: The uncertainty that exist in any analysis activity is represented here by three orthogonal dimensions: (a) the certainty of analysis, (b) the random variability, and (c) the type of variable. Each dimension has various categories, and the figure gives examples for each. The position of conventional engineering analysis methodologies is illustrated by the block. 
The abstraction uncertainty (or type of variable) describes whether the variable is quantitative or qualitative [6]. However, the distinction between these terms is loose, especially for "qualitative", so here the terms are defined by extending the four scales of Ackoff [7] to produce the six scales shown in Figure 2. Thus a 'quantitative' variable is either on a ratio (e.g. length) or interval (e.g. temperature $0^{\circ} \mathrm{C}$ ) scale.

Correspondingly, a 'qualitative' variable would be ordinal-numerical (e.g. Moh hardness of minerals), ordinal-textual (e.g. "hot, warm, cold"), nominal-numerical (e.g. jersey number on sport players), or nominal-textual (e.g. dishwasher soil types of "sauce, rice, jam").

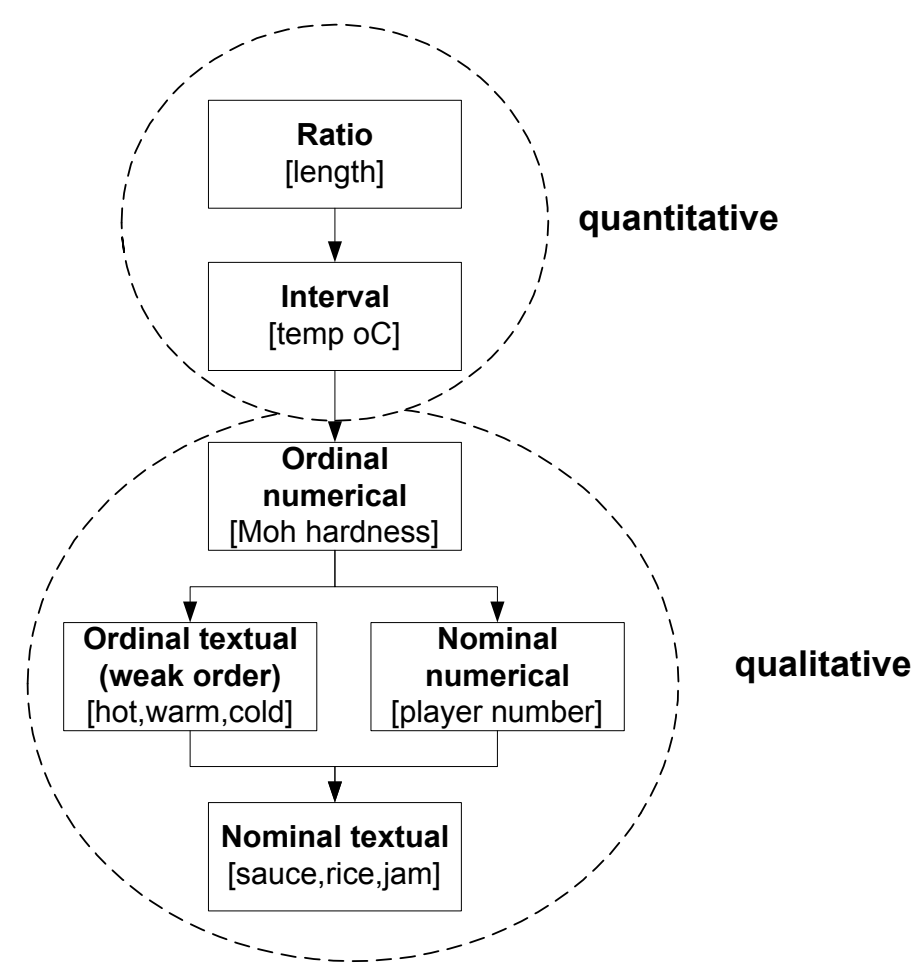

Figure 2: Classification system to distinguish between qualitative and quantitative variables. Examples of each type are given in brackets. The arrows indicate the direction of decreasing information content. 
A consequence of this structure is that no ordinal variable (even if numerical ) is considered quantitative. A variety of specialised design tools cover quantitative random variables, as described earlier [2]. Importantly, mathematical functions strictly only apply to quantitative variables. Unfortunately, this constraint is occasionally violated by designers when they enter an ordered variable into a mathematical calculation. This problem has previously been identified by Scott and Antonsson [8] as the 'weak order' problem. Some design methodologies, such as quality function deployment (QFD) and fuzzy theory, potentially suffer from this problem. To what extent a violation compromises the results is perhaps unknowable since there is no tool with perfect accuracy against which to compare. Nonetheless, it is professionally prudent that designers acknowledge the existence of uncertainty in output calculations when using ordered variables.

The uncertainty model could be extended: a fourth dimension being the need to repeat the simulation for each alternative design concept (each concept may have a different uncertainty profile), and a fifth dimension as time variant (dynamic) simulation.

Conventional engineering design calculations (engineering science) are shown in Figure 1 as being limited to explicit functions, no random variability (deterministic) and ratio (or interval) data types. However, engineering designers occasionally have to design with knowledge that is inadequate for system modelling (low certainty of analysis). Although research and development may increase certainty of analysis, this is usually at significant cost, and thus this remedy may not always be available to the Submitted to Journal of Engineering Manufacture (Proceedings of the Institution of Mechanical Engineers Part B) 
designer. Furthermore, there is often random variability in engineering problems, typically on quantitative variables in the form of manufacturing variability, or load-capacity interference.

Having established the types of uncertainty, the rest of this paper focuses on the methodologies for dealing with qualitative variables, and describes the DSI capabilities in this area.

\section{METHODOLOGIES FOR QUALITATIVE VARIABLES}

The typical problem with qualitative variables is that the certainty of analysis is usually also low. In addition, they may have random variability, which further compounds the problem. An example of such a variable is dishwasher Soil Type (Figure 5).

Established risk assessment methods of Monte Carlo analysis [9] and the Algebra of Random Variables [10] cannot accommodate this type of problem. Nor do engineering functional modelling systems address the problem, although there are possible exceptions such as prototypes using natural language fragments [11]. Quality function deployment (QFD) has some capabilities in this area as it transfers qualitative customer needs into a set of ranked engineering product attributes [12]. However, limitations include sensitivity of output importance to the number of variants of a characteristic that are initially listed, and the imposition of a numerical ranking on the customer requirements [13]. Also, QFD has no means to accommodate uncertainty in either the scoring or the relationships. Bayesian methods of probabilistic reasoning may be used to model quantitative, but not qualitative, uncertainty. The uncertainty has 
to be described explicitly by a probability distribution, which can be tedious, and it may be difficult to find a formal solution [14]. Bayesian methods require high certainty of analysis. Semi-quantitative simulation [15] uses numeric intervals to approximate ordinal numerical scales, producing quantitative outputs. Other qualitative scales are not accommodated. Fuzzy theory operates on ordered scales, both numerical and textual [16], accommodating the uncertainty in converting an ordinal scale to an interval one, which few other systems provide. However, fuzzy theory is limited to models with relatively high certainty of analysis (mathematical or logical relationships including expert system rules), and may not be used on nominal data types. Other methodologies that assign numbers to variables on ordered textual scales include genetic algorithms [17], utility theory, and multi-criteria decision analysis [18], where multiple aspects of utility (some of which may be ordered variables) are aggregated into a single measure of worth. They require high certainty of analysis, evident in the weighting function.

The methods described above either cannot operate on qualitative variables, or only on ordered variables, and cannot accommodate low certainty of analysis. Decision analysis [19] provides the most robust approach to modelling systems of (i) qualitative variables, (ii) with random variability, and (iii) low certainty of analysis. A limitation of the method is that it works best with variables that have only a few probably states, since it uses a combinatorial approach which can be tedious when there are many states to evaluate. Another limitation is the reliance on expert opinion, and while this is treated in a transparent way, it is necessarily subjective and therefore potentially contentious.

Submitted to Journal of Engineering Manufacture (Proceedings of the Institution of Mechanical Engineers Part B) 
The fact that there are limited options for modelling qualitative variables complicated the bigger objective of developing a methodology to handle both quantitative AND qualitative variables (as well as other types of uncertainty) in engineering design. The novel solution embodied in the Design for System Integrity (DSI) methodology $[1,2,3]$ was to use a discrete combinatorial [20] approach that is simultaneously compatible with the algebra of random variables (for quantitative variables and perfect knowledge) and decision theory (for qualitative variables and subjective knowledge). Importantly, the resulting methodology is also able to handle the cases intermediate to these two main processes. The quantitative process [3] is to take two input histograms and combine them in combinatorial manner using the given mathematical operator or decision table. The qualitative process within DSI is to use decision tables (termed maps) to determine an output qualitative variable (represented by a histogram) from two qualitative input variables (each represented by a histogram). The knowledge in the table is provided from expert opinion, and uncertain opinion at that.

DSI has been embodied in a software system that provides a user interface and encapsulates the algorithms. The software embodiment of DSI also includes catalogue features and multiple viewpoints, as support features for the engineering design environment. 


\section{OPERATION OF DSI}

The DSI process for qualitative variables is described by application to a subset of a model for wash performance of domestic dishwashers. A subsequent paper is intended to describe the broader context of the case study and the overall results.

\subsection{GENERAL OPERATING PRINCIPLES}

The sample problem is shown in Figure 3 (extracted from the DSI user interface) as a graph for determining Soil State from Soil Type and Soil Thermal Treatment using a decision table (map). Both Soil Type and Soil Thermal Treatment are uncertain textual variables and there is no necessity to interpret any order within them. The map that relates them to the Soil State is given in Figure 4. 


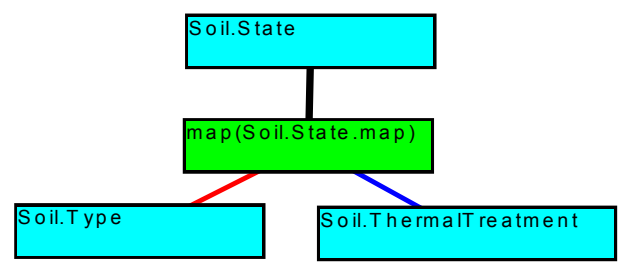

Figure 3: View of map operator in Design for System Integrity (DSI) software, showing that two inputs, Soil type and Soil thermal treatment are used to determine Soil state.

\begin{tabular}{|c|c|c|c|c|c|c|c|c|c|c|c|c|c|c|c|c|}
\hline Soil.State & & & Soil.Type & & & & & & & & & & & & & $\Delta$ \\
\hline & & & Clean & Bever & Sauce & Foodf & Rice & Peanut & Mashe & Egg & Oats & Break & Fat & Jam & Other & \\
\hline Soil. Therm & Fresh & Comment & & & & & & & & & & & & & & \\
\hline & & Clean & 1 & 0 & 0 & 0 & 0 & 0 & 0 & 0 & 0 & 0 & 0 & 0 & 0 & \\
\hline & & SolubleFilm & 0 & 0.333 & 0.1 & 0 & 0 & 0 & 0 & 0.5 & 0 & 0 & 0 & 0.5 & $0.142 \varepsilon$ & \\
\hline & & FineParticulate & 0 & 0.333 & 0.2 & 0.25 & 0 & 0 & 1 & 0 & 0.333 & 80.333 & 30 & 0 & 0.1428 & \\
\hline & & LoosePieces & 0 & 0 & 0.2 & 0.5 & 1 & 0 & 0 & 0 & 0.333 & 80.3333 & 30 & 0 & 0.1428 & \\
\hline & & StickyPaste & 0 & 0 & 0.3 & 0.25 & 0 & 1 & 0 & 0.5 & 0.333 & 30.333 & 30 & 0.5 & 0.1428 & \\
\hline & & Greasy & 0 & $0.333:$ & 0.2 & 0 & 0 & 0 & 0 & 0 & 0 & 0 & 1 & 0 & 0.1428 & \\
\hline & & DriedStuck & 0 & 0 & 0 & 0 & 0 & 0 & 0 & 0 & 0 & 0 & 0 & 0 & 0.1428 & \\
\hline & & CookedOn & 0 & 0 & 0 & 0 & 0 & 0 & 0 & 0 & 0 & 0 & 0 & 0 & 0.1428 & \\
\hline & & BurnedOn & 0 & 0 & 0 & 0 & 0 & 0 & 0 & 0 & 0 & 0 & 0 & 0 & 0 & \\
\hline
\end{tabular}

Figure 4: Portion of map to convert soil type and soil thermal treatment into soil state. Numbers are probabilities where each column sums to unity. 
For a given Soil Type (e.g. Sauce) and Soil Thermal Treatment (e.g. Fresh) the table shows the output Soil State and its variability in terms of probabilities (e.g. Soil State is 0\% probability of being Clean, 10\% Soluble Film, 20\% Fine Particulate, etc.). It is relevant to note that unlike the interval and fuzzy methods, the table does not need to contain mathematical expressions, nor is it necessary to transform the input variables to a numerical scale nor assume any order. The table simply expresses the belief system of an expert, and accommodates the uncertainty of that opinion by spreading the probability across multiple values (e.g. Soluble Film, Fine Particulate, etc. each get an allocation).

Once the knowledge in the map has been entered by the expert, and the model has been constructed using the graphical user interface in DSI, then it is time to run the system by asserting the values of the inputs. Here the Soil Type has been asserted to be as shown in Figure 5, and the Soil Thermal Treatment as in Figure 6, both of which are taken directly from the DSI user interface. The Soil Type distribution is based on the types and quantities of soils prescribed in the ANSI/AHAM [21] test for dishwashers. By asserting the inputs, the user is assigning probabilities, including zero probability, to the labels within the textual variables. If random variability were zero then the user would assign all the probability to one label, but in general will spread the total probability over multiple labels, as evident in Figure 5. 


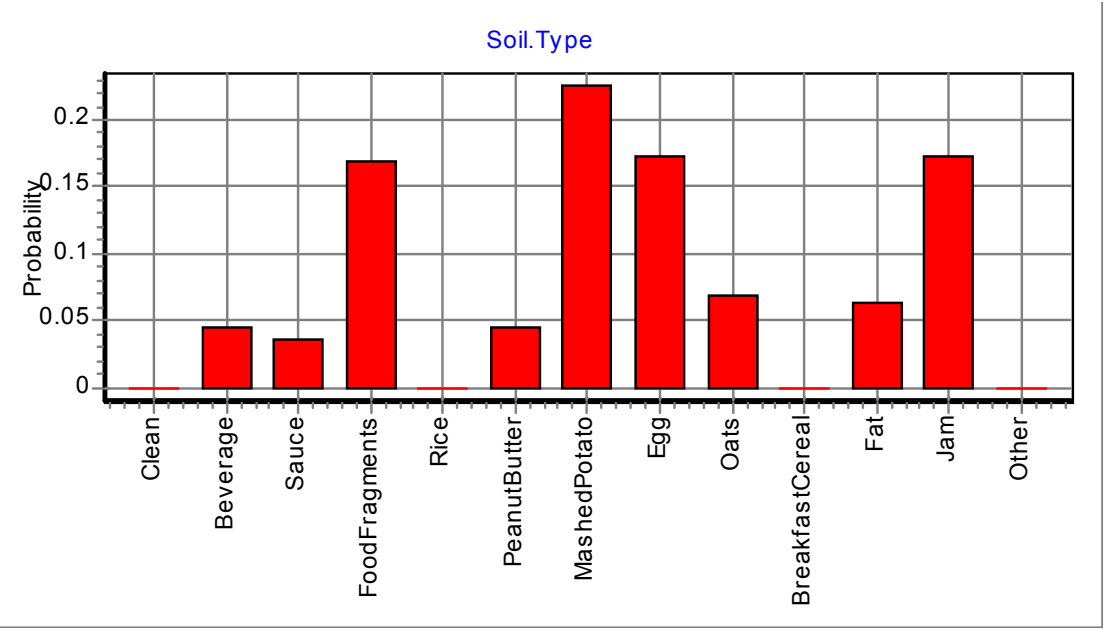

Figure 5: Soil type shown as a histogram based on mass of various soils used in the ANSI/AHAM test.

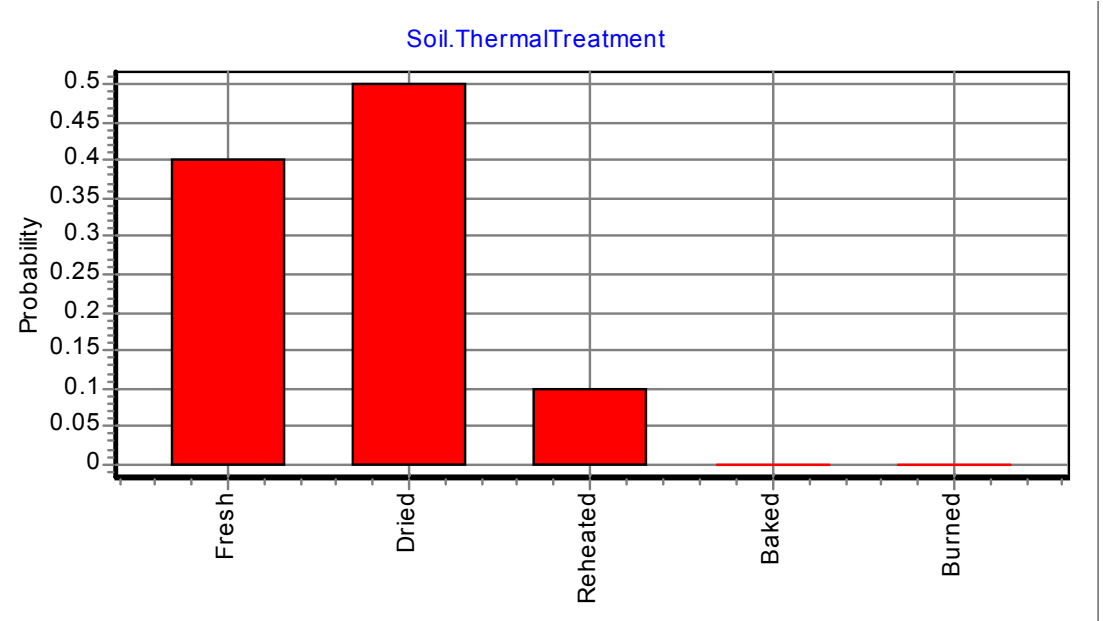

Figure 6: The profile for Soil thermal treatment can range from 'fresh' to 'burned' soil. For the ANSI/AHAM test there is only a single value: 'dried'. In this particular example it has also been asserted that some of the soil is 'fresh' and some 'reheated'. 
The output of the system is then calculated for each label of the output field. For example the probability of Soluble Film (within Soil State) is determined as the sum of the product of the assert probabilities and the respective map probability. The final DSI result is shown in Figure 7, as the uncertainty in the Soil State variable.

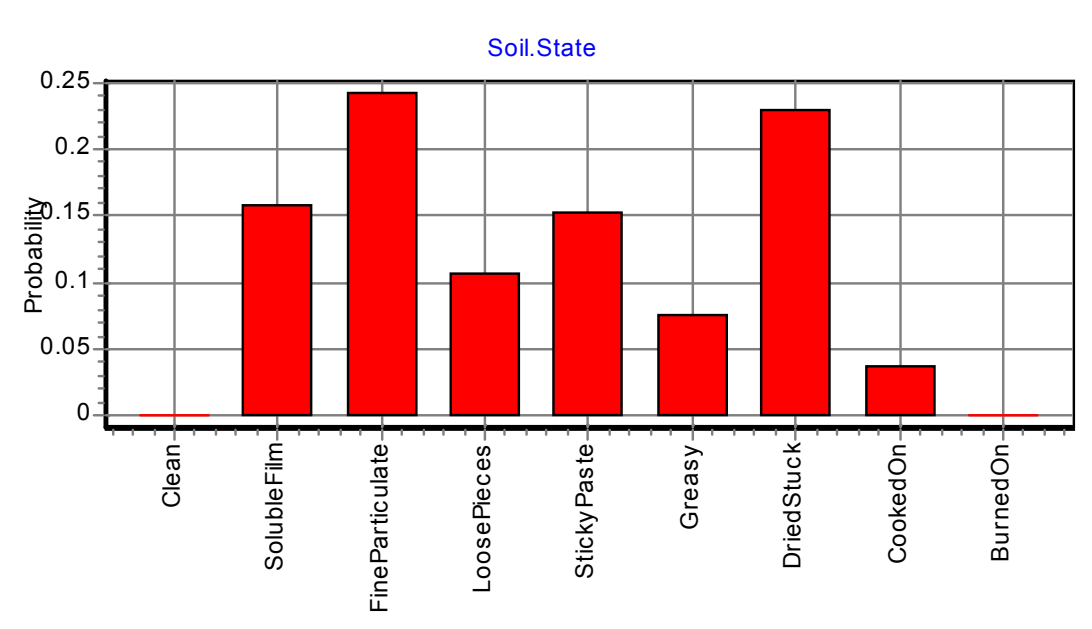

Figure 7: Soil state is a combination of soil type and soil thermal treatment. It describes the important wash characteristics of the soil.

The user may modify the profiles of the input assertions, e.g. set up another soil profile that includes more rice, to model the performance of the machine in a different market. In doing so the user reassigns the probabilities of the assertions only, and leaves the table probabilities unchanged. Alternatively, the table probabilities may be changed, but that corresponds to the expert changing the structure of the model. The table will have to be edited if one of the inputs gets a new qualitative item, for example if a new soil type 'honey' was added. If the table was not changed after such an insertion, then the DSI algorithm degrades gracefully by automatically creating an 'unresolved' label in the output to receive the probability contribution of the unexpected label. 


\subsection{DECREASING THE INFORMATION CONTENT}

It was stated above that DSI methodology is also able to handle cases of mixed variable type, and this is now demonstrated. In order to model some engineering systems it may be necessary to convert a higher order quantitative scale, (e.g. mass in kilograms: Figure 8), to a lower qualitative scale (e.g. an ordinal textual scale 'underweight, light, medium, heavy, overweight'). The process may be necessary when the distribution contains too much information to be useful or practical to process. This is readily accomplished with a DSI map (Figure 9) to produce the required textual description complete with uncertainty (Figure 10). 


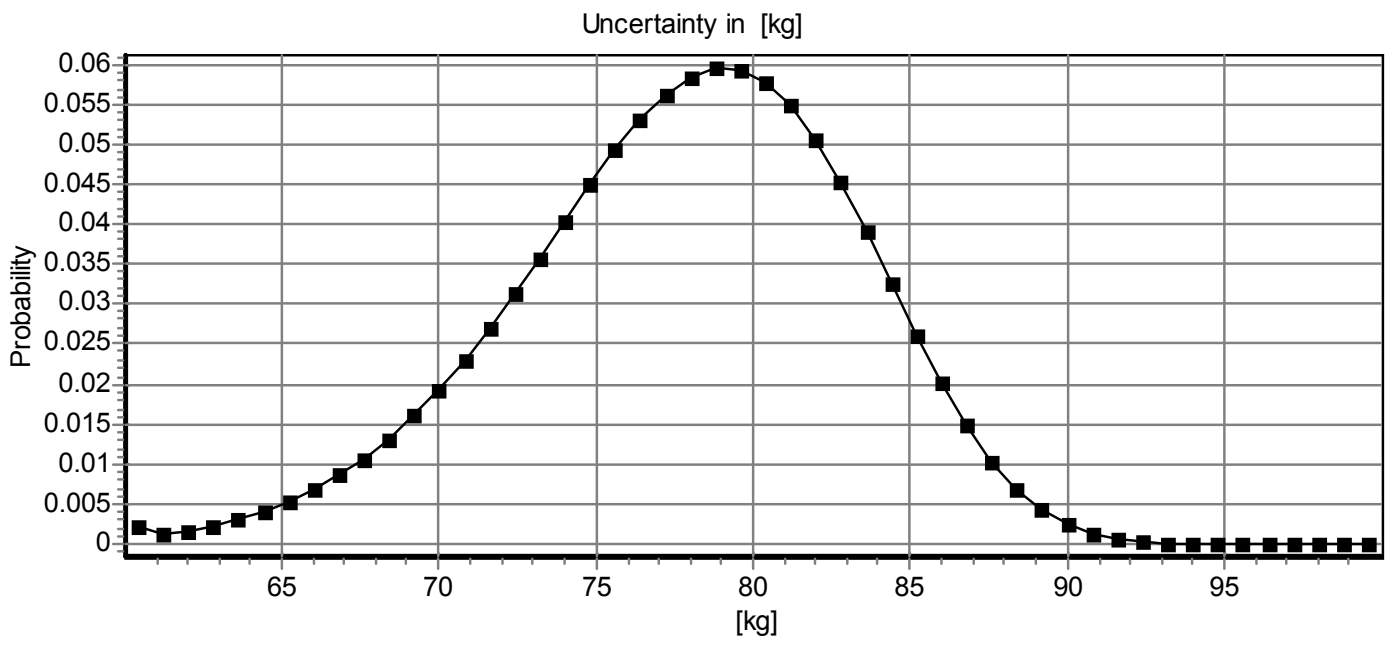

Figure 8: Random uncertainty in the variable 'Mass Quantitative' is expressed here as a probability distribution.

\begin{tabular}{l||l|l|l|l|l|l|l|l|l|}
\multicolumn{1}{l|}{ Integrity T } & & Mass & & & & & & & \\
\hline & & 50 & 60 & 70 & 80 & 90 & 100 & 110 & 120 \\
\hline A & Comment & & & & & & & & \\
\hline & Underweight & 0.8 & 0.2 & 0 & 0 & 0 & 0 & 0 & 0 \\
\hline & Light & 0.2 & 0.6 & 0.4 & 0.2 & 0 & 0 & 0 & 0 \\
\hline & Medium & 0 & 0.2 & 0.6 & 0.4 & 0.2 & 0 & 0 & 0 \\
\hline & Heaw & 0 & 0 & 0 & 0.4 & 0.6 & 0.2 & 0 & 0 \\
\hline & Overweight & 0 & 0 & 0 & 0 & 0.2 & 0.8 & 1 & 1
\end{tabular}

Figure 9: Map for converting Mass (50..120) into a qualitative variable ('Underweight ... Overweight'). The sum of each column sums to unity, and the analyst is free to distribute that total probability according to uncertain belief. 


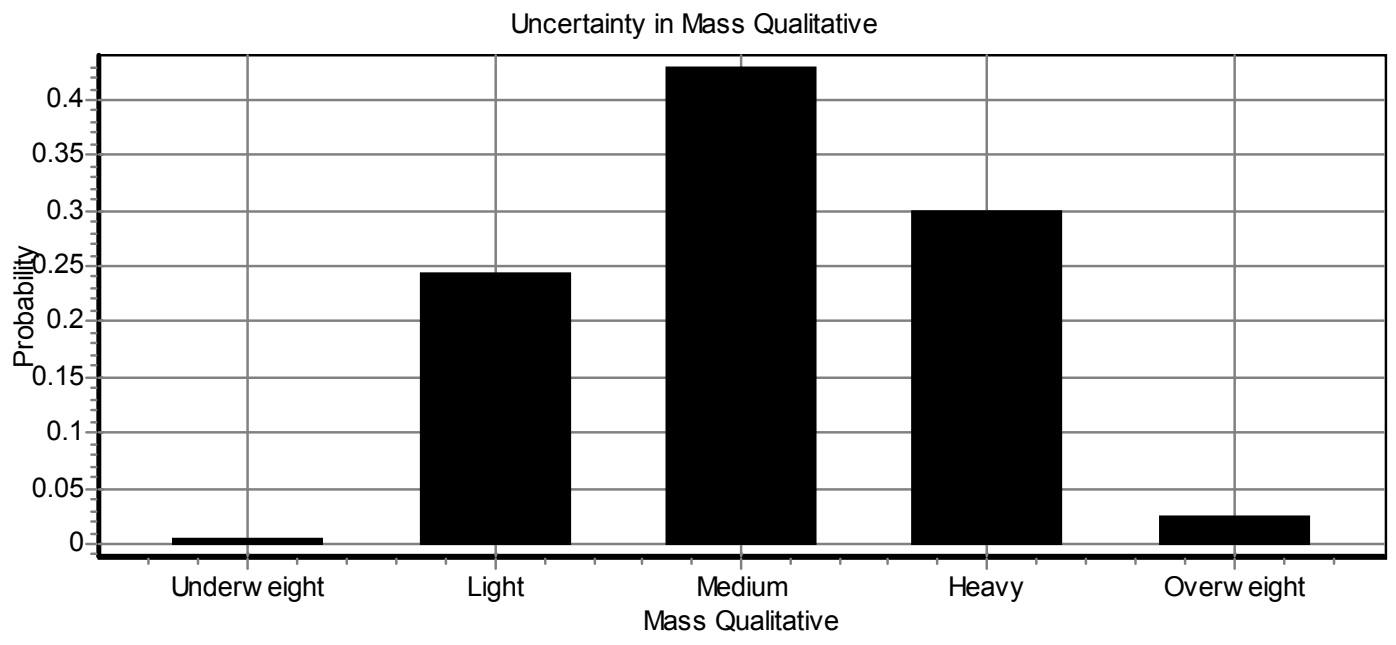

Figure 10: Qualitative output for mass, with uncertainty shown as a probability histogram.

From the above discussion on the 'weak order' problem, it is clear that the conversion of a single qualitative variable into a quantitative variable lacks robustness, although it is often done in design. However, it may be appropriate when the assignment is explicit [8]. The DSI maps provide just such an explicit assignment, in the form of stated beliefs (with their uncertainty). Even so, it is difficult from a purist perspective to see many cases where a designer or systems analyst can robustly make such a one-to-one conversion. However, the case seems stronger where multiple sources of qualitative Submitted to Journal of Engineering Manufacture (Proceedings of the Institution of Mechanical Engineers Part B) 
information are to be combined into a single quantitative output variable, again providing this was based on defendable knowledge. In the process a higher order variable is created, hence the information content is increased (though it is still uncertain). A DSI map provides such a mechanism and the process is illustrated in Figure 11, which is a fragment of a DSI model for dishwasher wash performance.
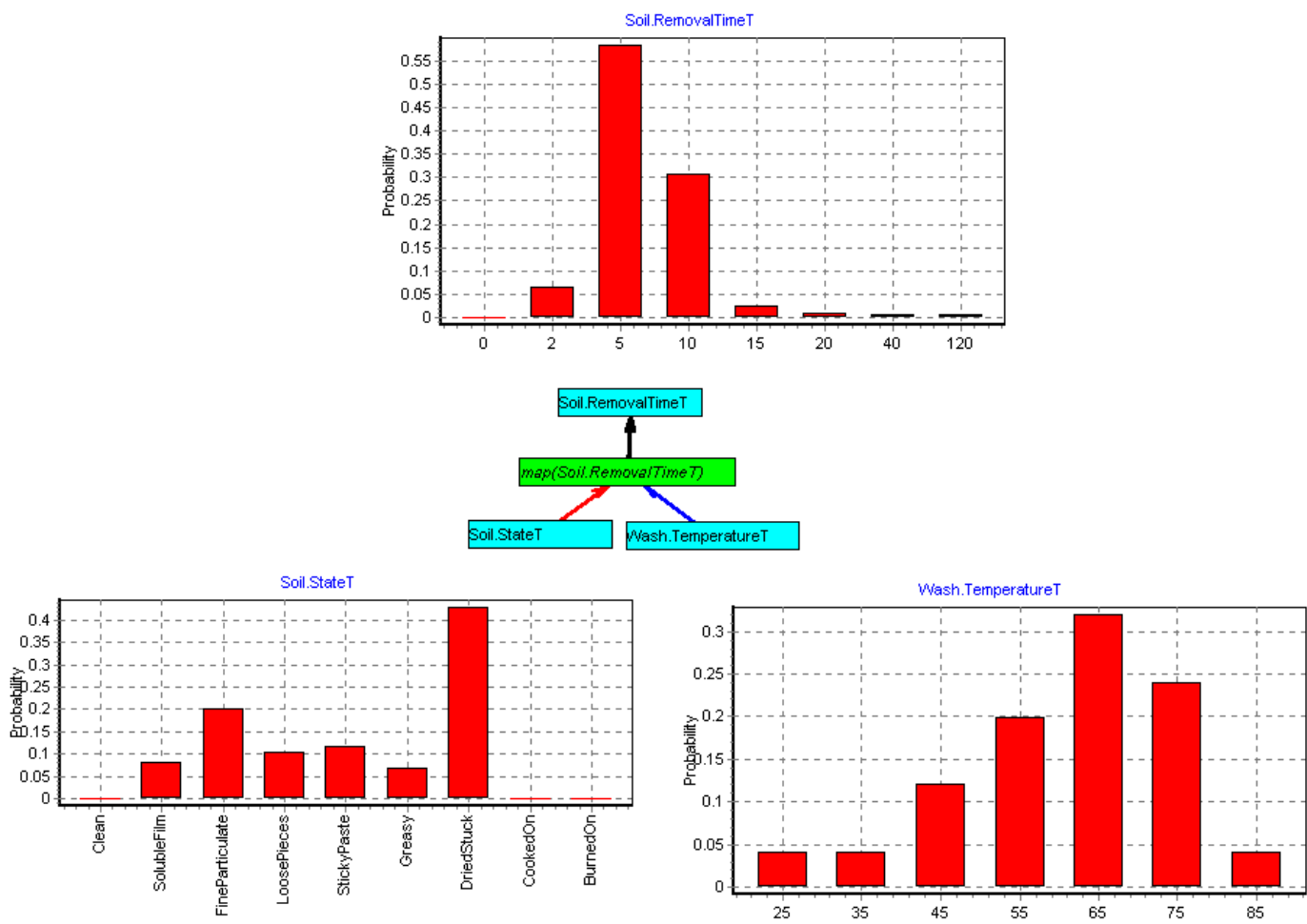

Figure 11: DSI model used to determine soil removal time using a map. The graph in the centre of the figure shows the operation. The inputs are the two distributions at the bottom of the figure, and the output is the top distribution. 
The input called SoilState $T$ is a variable describing the condition and type of soil on dishware that are placed in a dishwasher. It is qualitative: nominal textual since there is no ranking of the elements. The other input is Wash Temperature $T$ which is quantitative (interval scale), though it could be qualitative (nominal scale) if necessary. The map correlates these two inputs according to other knowledge, and uncertainty of that knowledge, that the expert has expressed in the map. The output of Soil removal time $T$ is a series of numbers which would be interpreted as quantitative (interval scale).

\subsection{CORRELATION TABLES}

Correlation tables are used (e.g. in Monte Carlo analysis) to correlate quantitative variables for which the usual assumption of independence does not hold. The DSI maps can be used for this purpose too. However the DSI maps are more powerful than conventional correlation tables as they:

(i) include uncertainty of the form 'if inputs are $A$ and $B$, then output is range $C_{i}$ with probabilities $p_{i}{ }^{\prime}$, and

(ii) they permit the inputs to be either quantitative or qualitative.

DSI maps permit all the various combinations of quantitative and qualitative variables to be used together in one model. Two of these cases have special names: two qualitative inputs producing a qualitative output is a decision table, and two quantitative inputs producing a quantitative output is a correlation table. The map methodology of DSI accommodates these two special cases as well as other ways of 
combining qualitative and quantitative data. DSI could be considered an extension of correlation tables towards qualitative variables, and equally as an extension of decision tables towards quantitative variables.

\section{DISCUSSION}

\section{Addressing the hypothesis}

Returning to the original hypothesis, the above excerpts demonstrate the qualitative capability of DSI, and together with the previous demonstration of quantitative function [2], show that DSI is indeed able to accommodate multiple types of uncertainty as might exist during engineering design. Benefits of the map approach of DSI are:

(1) The map provides a traceable and defendable record of the expert's opinion.

(2) The existence of uncertainty of analysis is acknowledged and formally represented in the map.

(3) The map accommodates all combinations of quantitative and qualitative variables.

(4) The map provides for uncertain correlation tables.

\section{Comparison with other methodologies}

The two obvious alternatives to DSI are decision theory and fuzzy theory. Compared to standard decision tables, DSI subsumes the functionality and extends beyond it to cope with fully quantitative variables. Where the DSI map process departs from conventional decision tables is in the ability to accept quantitative inputs, and to 
function as a correlation table. It also includes support for deviant variables (input labels that are not included in the decision table).

Compared to fuzzy theory, (i) DSI is not limited to ordinal textual data ('hot..cold'), but can use nominal data (non-ranked) data as well, and (ii) DSI is not limited to high certainty of analysis. Fuzzy theory transforms the variable onto an interval scale, solves the problem in the mathematical domain, and transforms the result back to an ordinal textual scale, whereas DSI solve the problem entirely in the qualitative domain without needing to transform the variables. Another significant difference (not necessarily a superiority) is that DSI provides a probabilistic computation consistent with both decision analysis, the algebra of random variables, and Monte Carlo analysis, whereas Fuzzy theory provides a possibilistic computation based on interval analysis.

The capabilities of decision theory and fuzzy theory are summarised in Figure 12, and of DSI in Figure 13. 


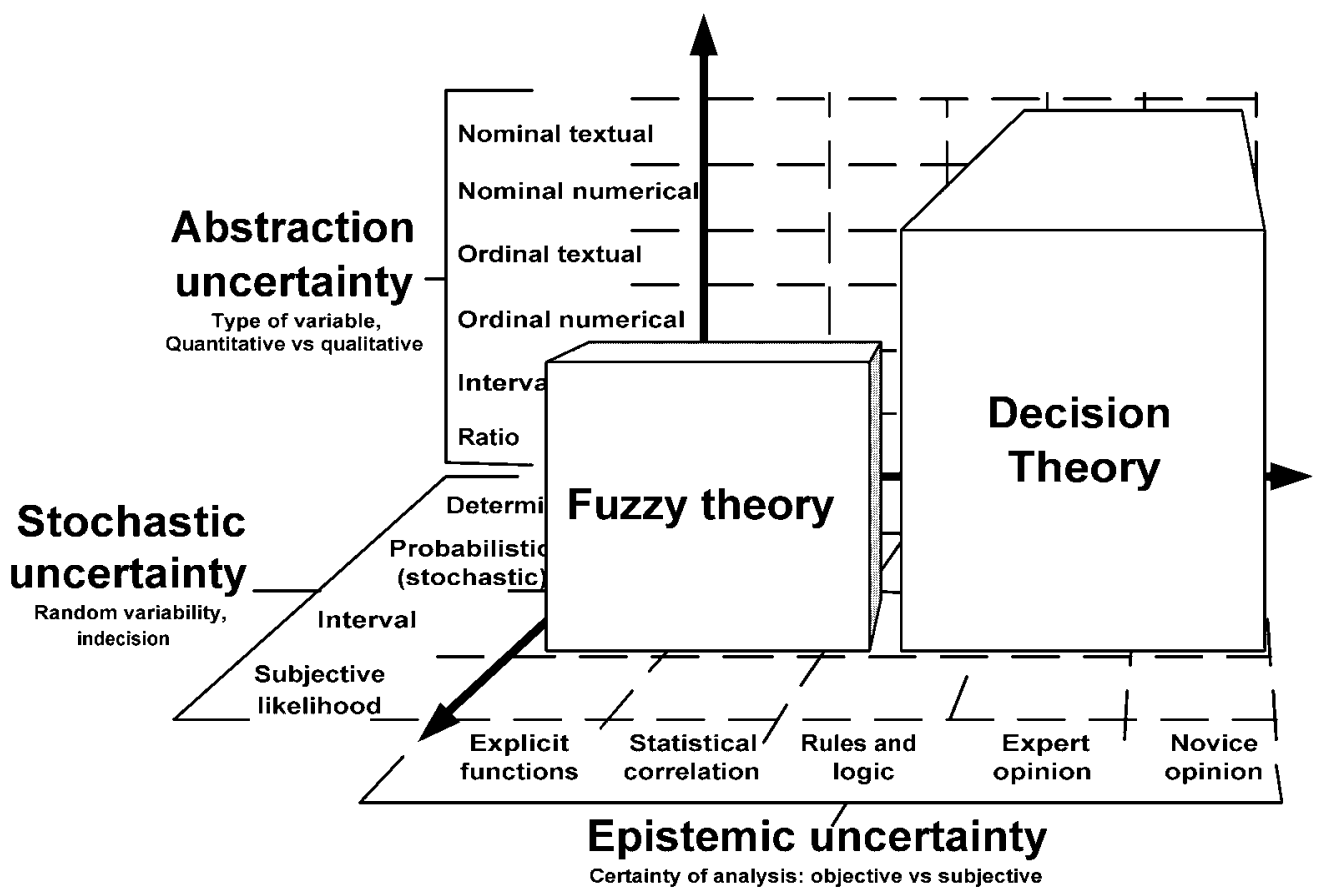

Figure 12: Fuzzy theory and decision theory have different capabilities on the three dimensions of uncertainty. 


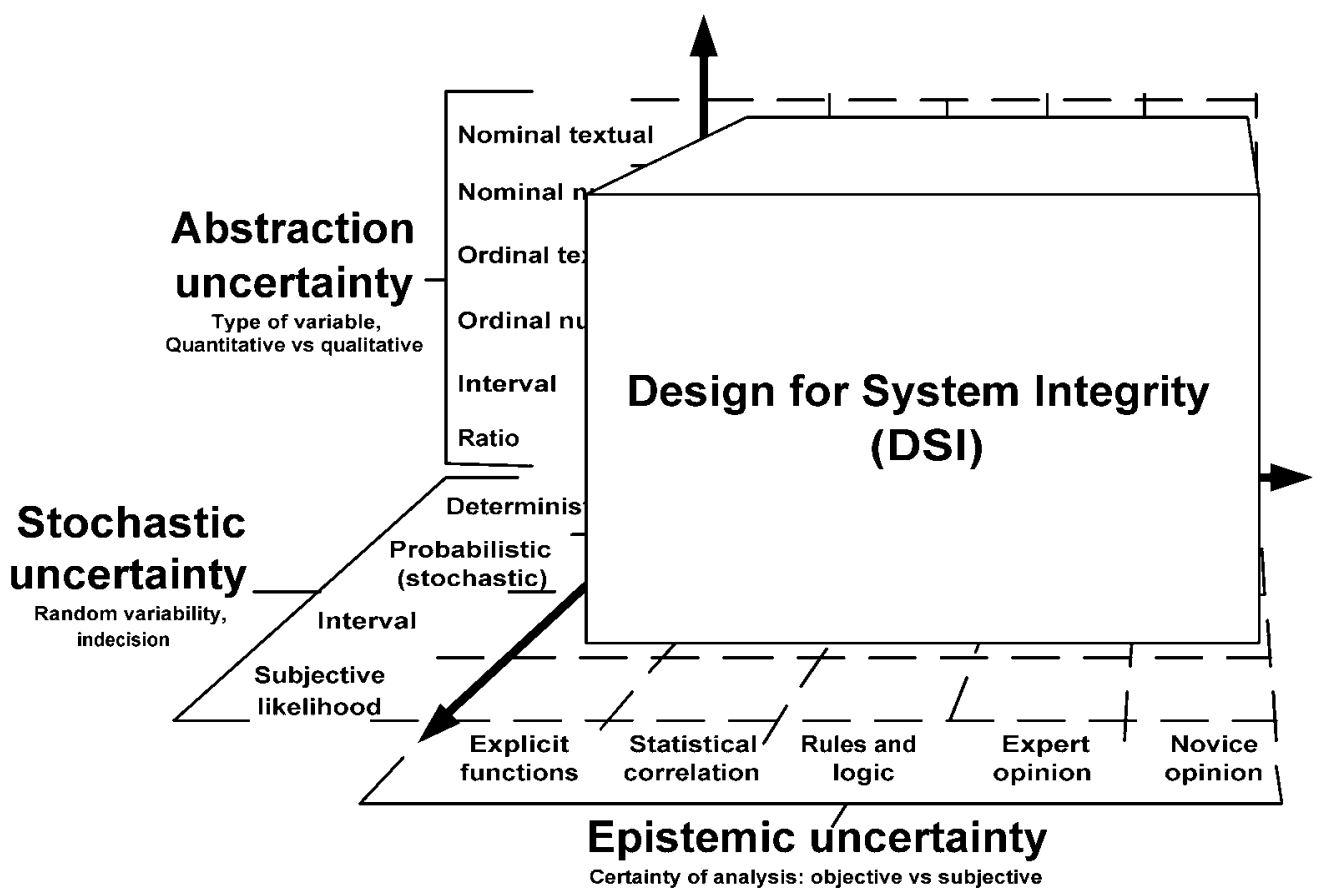

Figure 13: Capabilities of DSI on the three dimensions of uncertainty.

\section{Limitations of DSI}

The DSI decision tables can model problems that can be formulated as two uncertain input variables that subjectively or objectively determine an output variable. It does however require probabilities of those input variables, and will not accommodate subjective likelihood (e.g. "highly unlikely"). It is also dependent on expert opinion, which naturally is always subjective even if informed. This is possibly unavoidable, and common to other established methodologies in risk assessment. Useful results may nonetheless be obtainable. When the results of the analysis are used only within the organisation, then subjectivity may not be an issue as an analysis with imperfect 
information will often provide more of a competitive advantage than no analysis, and the expert usually has a relationship of acceptance with the organisation. This situation is typical of engineering design. However, in the larger risk assessment environment these provisos may not hold, especially where other protagonists (e.g. the public or the courts) may question the validity of the expert opinion. The work therefore connects with risk assessment [22] and risk perceptions [23], though these interesting topics are beyond the scope of the present paper.

\section{$6 \quad$ CONCLUSIONS}

Following earlier papers in the series [2,3], this paper presented the Design for System Integrity (DSI) methodology, especially its processes for qualitative variables. It was shown that a modelling system could be developed to accommodate the multiple types of uncertainty that can exist during engineering design, including the processing of qualitative variables through imperfectly understood systems. To clarify the problem, the paper identified several independent dimensions of uncertainty. The abilities of existing methodologies to accommodate qualitative variables were reviewed. The principles of DSI were explained, particularly its ability to operate on uncertain textual non-ordered variables given subjective and imperfect knowledge (including uncertain opinion). DSI has the ability to downgrade the information content in a variable, where the primary variable contains too much information to be practical. It can also upgrade the information content of a variable (e.g. an ordinal variable to an interval one), and the conditions under which this is valid were discussed. Conceptually, DSI combines the functionality of correlation tables and decision tables, and is able to operate on both 
quantitative and qualitative variables in the same model.

The originality in the work is that DSI accommodates multiple types of uncertainty, namely certainty of analysis, random variability, and type of variable, within a single methodology. These multiple forms of uncertainty appear in the engineering design process, and the value of DSI is consequently its support for decision making under uncertainty, i.e. risk management in engineering design.

It is intended that a future paper will describe the application of the DSI methodology to a case study in engineering design.

\section{Acknowledgement}

We gratefully acknowledge the involvement of our late colleague Dr Ken Whybrew (University of Canterbury, 2001) in the co-supervision of Dirk Pons' doctoral research, on which this paper is based. 


\section{References}

1 Pons D.J. A methodology for system integrity in design. PhD thesis, 2001, University of Canterbury, Christchurch, New Zealand.

2 Raine J.K., Pons D.J., and Whybrew K. The design process and a methodology for system integrity. Journal of Engineering Manufacture (Proceedings of the Institution of Mechanical Engineers Part B), 2001 Vol 215, p569-576.

3 Pons D.J., and Raine J.K. Relative effectiveness of mechanisms for simulating uncertainty in quantitative systems. Journal of Engineering Manufacture (Proceedings of the Institution of Mechanical Engineers Part B), 2003, in press.

4 D'Ambrosio B., and Ullman D. Decision problem representations for collaborative design. http://cs.oregonstate.edu/ dambrosi/ijcaibpm.ps.Z, downloaded from http://www.cs.orst.edu/ dambrosi/edss/info.html, 1995, last updated 19 April 1996 and accessed on 23 Jan 2001

$5 \quad$ Biondo S.J. Fundamentals of expert systems technology, 1990, Ablex Publishing, New Jersey 
6 Ullman D., and D'Ambrosio B. A taxonomy for classifying engineering decision problems and support systems. ASME Design Engineering Division (Publication) DE, 1995, v83 n2 Pt1 p627-637.

7 Ackoff R.L. Scientific method optimising applied research decisions, 1962, John Wiley \& Sons, New York.

8 Scott M.J., and Antonsson E.K. Arrow's Theorem and engineering design decision making. Research in Engineering Design - Theory, Applications, and Concurrent Engineering, 1999, 11, 4 p 218-228.

9 Vose D. Quantitative risk analysis, 1996, (John Wiley), ISBN 0-471-95803-4.

10 Springer M.D. The algebra of random variables, 1979, John Wiley \& Sons, New York, ISBN 0-471-01406-0.

11 Deng Y-M., Britton G.A., and Tor S.B. Design perspective of mechanical function and its object-oriented representation scheme, Engineering with Computers, 1998, v 14 n 4 p 309-320

12 Gustafsson A. Customer focused product development by conjoint analysis and QFD, 1996, Linköping Studies in Science and Technology, Dissertation No. 418, Division of Quality Technology, Department of Mechanical Engineering, Linköping University, S-581 83 Linköping Sweden 
13 Mill H.F. Simplifying the implementation of QFD, IEE Colloquium (Digest), 1994, n 086 May 6 p 5/1-5/4

14 Siu N. Monte Carlo method for multiple parameter estimation in the presence of uncertain data, Reliability Engineering \& System Safety, 1990, v 28 n 1 p 59-98

15 Berleant D., and Kuipers B.J. Qualitative and quantitative simulation: bridging the gap, Artificial Intelligence, 1997, v 95 n 2 Sep p 215-255

16 Wood K.L., Antonsson E.K., and Beck J.L. Comparing fuzzy and probability calculus for representing imprecision in preliminary engineering design. American Society of Mechanical Engineers, Design Engineering Division (Publication) DE, 1989, v 17 Sep 17-21 p99-105.

17 Santillan-Gutierrez S., and Wright I. Solution clustering with genetic algorithms and DFA: an experimental approach, In SHARPE J (ed), A I system support for conceptual design, Proceedings of the 1995 Lancaster International Workshop on Engineering Design, 1996, Springer: p37-53

18 Taylor B.W. Introduction to management science, 1999, Sixth edition, Prentice Hall, New Jersey USA, ISBN 0-13-918103-2

19 Clemen R.T. Making hard decisions, 1996, 2e, Duxbury Press Submitted to Journal of Engineering Manufacture (Proceedings of the Institution of Mechanical Engineers Part B) 
20 Cooper D.F., and Chapman C.B, Risk analysis for large projects. 1987, John Wiley \& Sons, ISBN 0-471-91247-6

21 ANSI/AHAM DW-1-1992 Household electric dishwashers, 1992, American National Standard, Association of Home Appliance Manufacturers, 20 North Wacker Drive, Chicago, Illinois 60606

22 Bley D., Kaplan S., and Johnson D. Strengths and limitations of PSA: Where we stand. Reliability Engineering \& System Safety, 1992, v 38, p 3-26

23 Jasanoff S. The political science of risk perception. Reliability Engineering \& System Safety, 1998, 59 (1): p91-99 


\section{List of captions}

Figure 1: $\quad$ The uncertainty that exist in any analysis activity is represented here by three orthogonal dimensions: (a) the certainty of analysis, (b) the random variability, and (c) the type of variable. Each dimension has various categories, and the figure gives examples for each. The position of conventional engineering analysis methodologies is illustrated by the block.

Figure 2: $\quad$ Classification system to distinguish between qualitative and quantitative variables. Examples of each type are given in brackets. The arrows indicate the direction of decreasing information content.

Figure 3: $\quad$ View of map operator in Design for System Integrity (DSI) software, showing that two inputs, Soil type and Soil thermal treatment are used to determine Soil state.

Figure 4: $\quad$ Portion of map to convert soil type and soil thermal treatment into soil state. Numbers are probabilities where each column sums to unity.

Figure 5: Soil type shown as a histogram based on mass of various soils used in the ANSI/AHAM test.

Figure 6: $\quad$ The profile for Soil thermal treatment can range from 'fresh' to 'burned' soil. For the ANSI/AHAM test there is only a single value: 'dried'. In this particular example it has also been asserted that some of the soil is 'fresh' and some 'reheated'.

Figure 7: Soil state is a combination of soil type and soil thermal treatment. It describes the important wash characteristics of the soil.

Figure 8: $\quad$ Random uncertainty in the variable 'Mass Quantitative' is expressed here as a probability distribution.

Figure 9: $\quad$ Map for converting Mass (50..120) into a qualitative variable ('Underweight ... Overweight'). The sum of each column sums to unity, and the analyst is free to distribute that total probability according to uncertain belief.

Figure 10: Qualitative output for mass, with uncertainty shown as a probability histogram.

Figure 11: $\quad$ DSI model used to determine soil removal time using a map. The graph in the centre of the figure shows the operation. The inputs are the two distributions at the bottom of the figure, and the output is the top distribution.

Figure 12: Fuzzy theory and decision theory have different capabilities on the three dimensions of uncertainty.

Figure 13: Capabilities of DSI on the three dimensions of uncertainty. 